DUO LI, Ph.D. ${ }^{1}$

(Corresponding author)

E-mail: duoli0725@gmail.com

PRAKASH RANJITKAR, Ph.D. ${ }^{2}$

E-mail: p.ranjitkar@auckland.ac.nz

YIFEI ZHAO, Ph.D. ${ }^{1}$

E-mail: 409291742@qq.com

1 Highway School, Chang an University

Xi an 710000 , China

2 Department of Civil \& Environmental Engineering

University of Auckland

Private Bag 92019, Auckland 1142, New Zealand
Intelligent Transport Systems (ITS)

Preliminary Communication

Submitted: Dec. 23, 2015

Accepted: Apr. 12, 2016

\title{
EFFICIENCY AND EQUITY PERFORMANCE OF A COORDINATED RAMP METERING ALGORITHM
}

\begin{abstract}
Ramp metering (RM) has been widely applied due to its effectiveness in improving motorway traffic conditions by limiting inflow from on-ramps. A great deal of experimental and simulation-based studies have proven system-wide benefits of motorways from RM. Benefits attributed to RM in the literature include reducing travel times, increasing motorway throughputs and decreasing fuel consumption and emissions. However, RM benefits might be costing more some motorway users, e.g. some on-ramp users might be experiencing longer delay than others, which leads to an unfair allocation of RM benefits. This paper presents a coordinated ramp metering strategy, which is aimed at reducing the inequity among motorway users using different on-ramps and investigates trade-offs between efficiency and equity for the proposed strategy. Total travel time is used to measure the efficiency while Gini coefficient is used to measure equity. A combined index is proposed incorporating the two measures to serve as an objective function to solve the bi-objective control design problem. The performance of the proposed strategy is verified by comparing it to a well-established coordinated ramp metering strategy HERO using micro-simulation software AIMSUN. Simulation results revealed that the equity of the motorway system can be improved significantly by using the proposed strategy without compromising much on the efficiency of the system.
\end{abstract}

\section{KEY WORDS}

ramp metering; efficiency; equity; AIMSUN;

\section{INTRODUCTION}

Ramp metering (RM) is an efficient traffic control tool in allocating limited motorway capacity. RM has been widely applied in many countries due to its effectiveness in improving motorway traffic conditions by limiting inflow from on-ramps. A great deal of experimental and simulation studies conducted in the past have shown system-wide benefits of motorways from RM. RM exerts effects on efficiency and equity performance of motorways. Efficiency benefits attributed to RM in literature [1-4] include reducing travel times, increasing motorway throughputs and decreasing fuel consumption and emissions. Travel time and throughput reflect mobility and capacity of a motorway system, respectively. However, efficiency benefits might be obtained at cost of some individuals, leading to an unfair allocation of benefits among motorway users. The equity issue in RM control system was raised in literature as early as in the 1960s [5]. Yin et al. [6] stated that the lack of consideration of inequity among road users has adversely influenced public acceptance and hindered the widespread use of RM. Zhang and Levinson [1] suggested that although improving efficiency of motorway systems is the original and still the most important objective of RM, user equity should be considered at least as the secondary goal to make it a viable system.

The concept of equity is broad and ambiguous. Absence of explicit definition and measurement of equality might be the main reason that user equality has not been sufficiently considered in RM studies [6]. Levinson et al. [7] introduced Gini coefficient [8], a widely accepted measure in economic studies to the field of RM. Two equality measures were defined including spatial equity and temporal equity. The former reflects the distribution of delays among drivers using different on-ramps at the same time while the latter measures inequity among drivers using the same onramp at different time. As a spatial equity measure is suitable to capture the aforementioned issue of RM, hereafter in the paper, "equity" will be used to refer to "spatial equity".

Kotsialos and Papageorgiou [9] observed a partially conflicting relationship between efficiency and equity, or in other words, a trade-off between the two. Zhang and Levinson [10] reported that the most efficient RM strategy is the least equitable one. However, 
in a more recent study, Zhang and Shen [11] stated that the objectives of equity and efficiency can be obtained simultaneously and they do not necessarily conflict with each other.

Traffic responsive RM strategies can be classified into two groups, namely: local ramp metering (LRM) and coordinated ramp metering (CRM). The former determines the metering rate based on local traffic conditions whereas the latter utilizes system-wide traffic measurements from an entire region of the network to control all on-ramps within that region. CRM outperforms LRM when addressing multiple bottlenecks, limited on-ramp storage spaces and inequity to motorway users. In CRM, optimal control-based strategies employ relatively complicated numerical solution algorithms, making it quite challenging to implement in the field. This might be the main reason that most of CRM strategies operated in the field are heuristic rule-based strategies. Papamichail and Papageorgiou [12] proposed a HEuristic Ramp metering coOrdination (HERO) strategy, which claims to have achieved efficiency close to that of some sophisticated optimal control strategies. Besides, it is a simple and transparent strategy that does not require any model to predict external disturbances. HERO strategy is in operation on Motorway A6 in Paris, France and Monash Freeway in Melbourne, Australia. Papamichail et al. [13] performed a before and after study of Monash Freeway and revealed efficiency gains using HERO strategy. However, the study did not touch on equity aspect of the strategy. Stratified ZONE RM strategy addressed the equity issue through imposing maximum queue constraints that ensure delay less than four minutes per vehicle on local ramps and less than two minutes per vehicle on motorway to motorway ramps [14].

More recently, Li and Ranjitkar [15] demonstrated that HERO when integrated properly with variable speed limit (VSL) is more effective to distribute delay among different on-ramps in the CRM system. VSL has proven to be an effective ITS measure to achieve significant mobility gains [16]. In HERO, the minimum queue length assigned to each upstream on-ramp is a proportion of the maximum admissible queue length of the respective on-ramp. This implies that in a group of coordinated on-ramps with varying length of onramps, vehicles using longer on-ramps having larger storage space might experience relatively longer delay compared to shorter on-ramps, hence leading to an unfair system.

This paper proposes a new coordinated ramp metering strategy aimed at reducing the inequity among motorway users using different on-ramps and investigates trade-offs between efficiency and equity for the proposed strategy. Total travel time is used to measure efficiency while Gini coefficient is used to measure equity. A combined index is proposed incorporating the two measures to serve as an objective function to solve the bi-objective control design problem. The performance of the proposed strategy is verified by comparing it against HERO strategy using micro-simulation software AIMSUN.

\section{EQUITY IN RAMP METERING}

A newly designed or an updated control system would have two effects, namely, the "generative" effect and the "distributive" effect [17]. The former refers to the social welfare improvements resulting from the investment in transportation systems, while the latter occurs when some of the positive effects are compensated for by the negative ones. In traffic control systems, the generative effect and distributive effect are equivalent to the efficiency performance and the equity performance, respectively. The efficiency of RM in improving motorway throughputs, environmental quality and travel time savings have been verified by a number of experimental and simulation studies (e.g. [18-19]). However, system-wide efficiency benefits of RM might be obtained at the cost of some motorway users e.g. some on-ramp users might be experiencing longer delay than others, which can lead to an inequitable distribution of benefits from RM.

As mentioned earlier in the introduction, the equity of traffic control strategies is a broad and ambiguous concept. Kesten et al. [20] mentioned that the indicators used in the literature to measure equity of traffic control strategies are mainly adapted from statistical or socio-economic models. The former examines the distribution of any variable in a given population while the latter is based on welfare economics and integrates equity concerns into a welfare function. Examples of statistical measures are: range, variance, measure of variation, log variance, Gini measure and Theil's entropy measure. The axiomatic measures can be used to assess the inequality of any vector or distribution of observations.

Some operational RM strategies, e.g. Bottleneck algorithm [22], have employed a queue override control to decrease the user inequality. Similarly, Stratified Zone algorithm [14] has a maximum ramp delay constraint, which ensures the delay of each vehicle is less than four minutes at the on-ramp. In fact, the minimum metering rates included in a number of RM systems make contribution to the equity as well. Nevertheless, their influence is not easy to determine in advance so the balancing process is obtained implicitly [21].

Kotsialos and Papageorgiou [23] addressed the equity issue by imposing different on-ramp storage restrictions in an optimal control based Advanced 
Motorway Optimal Control (AMOC) strategy. They observedatrade-offbetween efficiencyandfairness, which the AMOC strategy addressed implicitly with consideration of the available on-ramp storage space. Zhang and Levinson [1] proposed a RM objective function to minimize total weighted travel time, which can be used to balance efficiency and equity of RM control via weighting on-ramp delays nonlinearly. Nevertheless, they acknowledged that it was difficult to obtain an effective weighting function for on-ramp delays.

Gini Coefficient [8] and the associated Lorenz Curve [24] have been borrowed in the field of RM to measure the inequity due to RM. Yin et al. [6] used Gini coefficients to analyze trip cost ratios before and after applying RM in a southern California corridor. In Twin Cities RM shut-off experiment [25], the Lorenz Curve was plotted with the absolute terms of travel delay for motorway users.

Minimizing the overall travel cost for road users is overwhelmingly the most important goal for most motorway management systems. Only recently the equity aspect has become a concern. User equity has emerged as an important criterion for control practices. Explicitly or implicitly, the two aspects have been considered as competing criteria [23]. However, Yin et al. [6] implied that balancing efficiency and equity in one motorway system is not necessarily a zero-sum game. Therefore, it is reasonable to adjust the existing strategy to be more publicly acceptable, equitable and efficient via better design of control systems to fully grasp the working mechanism between different control objectives.

\section{HERO STRATEGY}

Papamichail and Papageorgiou [13] proposed HERO as a rule-based CRM strategy that incorporates ALINEA at the local level in a group of on-ramps within the control string. A control string contains several local RM controllers and a central controller that is used to communicate with and coordinate each local controller. ALINEA is a closed-loop feedback RM strategy, in which the metering rate is determined in proportion to the difference between the desired occupancy and observed downstream occupancy [26].

$r(k)=r(k-1)+K_{R}\left[\hat{O}-O_{\text {out }}(k)\right]$

where: $K_{R}(>0)$ is a regulator parameter with a recommended value of $70 \mathrm{veh} / \mathrm{h}, \hat{O}$ is a desired set value for the downstream occupancy, $O_{\text {out }}(k)$ is the observed downstream occupancy, and $r(k-1)$ is the metering rate at $k$-1 time step.

At network level, when the queue length at any onramp exceeds a pre-determined threshold value, HERO assigns it as a master on-ramp and then gradually employs successive upstream on-ramps to work as slave ramp meters. The maximum slaves number should be pre-defined to avoid an unnecessary delay at the metered slave on-ramp. A maximum number of 4-6 slave on-ramps may be suitable for many application cases. This strategy exploits storage spaces of upstream on-ramps to enlarge the storage capacity of the critical bottleneck by maintaining the minimum queue lengths at slave on-ramps. The working principle of the HERO strategy as proposed by its developers can be outlined as follows:

1) At each control time interval $T_{c}$, HERO controllers receive from local controllers the information on current on-ramp queue length and traffic states of the mainline; based on which it decides a possible coordination action.

2) Whenever a relative on-ramp queue length exceeds a pre-specified activation threshold value, HERO control strategy is activated and the respective onramp is turned as a "master". The "master" gradually employs successive upstream on-ramps as "slaves".

3) HERO assigns the desired minimum queue lengths to the successive upstream on-ramps to avoid long queues at the "master" on-ramp. The desired minimum queue length $W_{\text {min }}(k)$ is computed as follows:

$W_{\min }(k)=\frac{W_{\max }(k) \cdot \operatorname{Sum} W(i)}{\operatorname{Sum} W_{\max }(i)}$

where: $W_{\max }(k)$ is the maximum admissible queue length of the respective on-ramp, Sum_W(i) is a sum of current queue lengths at each on-ramp within the coordination control string, and $S u m_{-} W_{\max }(i)$ is a sum of the maximum admissible queue lengths at each on-ramp within the coordination control string.

4) For each $T_{c}$, HERO updates the desired minimum queue lengths at each "slave" on-ramp so that the relative queue lengths at each on-ramp can be kept close to each other.

5) HERO gets deactivated when the relative queue length of the "master" on-ramp drops below the deactivation threshold value.

\section{MODIFIED HERO STRATEGY}

As discussed earlier in the introduction, HERO assigns the minimum queue length to each upstream on-ramp in a proportion of the maximum admissible queue length of the respective on-ramp using Equation 2, which can lead to unfair distribution of delay for different on-ramp users. To address the equity issue, we propose to replace Equation 2 with the following equations that will ensure fairer distribution of delay among different on-ramp users:

$$
\begin{aligned}
& W_{\min }(k)=\min \left\{W_{1}(k), W_{2}(k)\right\} \\
& W_{1}(k)=\text { Sum_W(i)/N } \\
& W_{2}(k)=W_{\max }(k) \cdot a
\end{aligned}
$$


where: $a$ is a control parameter ranging from 0 to 1 , and $N$ is the number of on-ramps within the coordination control string.

Here, $W_{1}(k)$ maintains the relative queue lengths at each on-ramp close to each other while $W_{2}(k)$ prevents queues at on-ramps from interfering with the adjacent street traffic. By selecting the minimum of the two values, the minimum desired queue length at each onramp is determined.

Figure 1 presents a flowchart of the proposed strategy, which works in three different steps. In the first step, a master on-ramp is detected once the observed queue length exceeds a pre-specified queue length threshold, which is equal to the product of act ( $j$ ) and $W_{\max }(i)$. This step is related to the working principle \#2 of the HERO strategy as described in the previous section. The second step is to define dissolution of the coordination string, which is related to the working principle \# 5 of the HERO strategy. In the third step, the minimum queue length is determined for each "slave" on-ramp using Equation 3, which is related to the working principle \# 4 of the HERO strategy. The minimum queue length computed using Equation 3 is maintained at each "slave" on-ramp using the following formulation.

If $w(k)>W_{\min }(k)$ then,

$R(k)=r^{\prime}(k)$

Otherwise,

$R(k)=\min \left\{r(k), r^{-}(k)\right\}$

$r^{\prime}(k)=\frac{1}{T}\left[w(k)-W_{\min }(k)\right]+d(k-1)$

where: $R(k)$ is the metering rate to be applied at the time step $k, r(k)$ is the metering rate determined by ALINEA strategy, $r^{\prime}(k)$ is the metering rate used to maintain the desired queue length, $T$ is the time interval, $w(k)$ is the current queue length, $d(k-1)$ is the demand flow entering the on-ramp.

Equation 6 and Equation 7 will help to maintain the on-ramp queue length close to the desired minimum queue length $W_{\text {min }}(k)$ assigned by the proposed CRM strategy to each "slave" on-ramp. Equation 8 will yield a lower $r^{-}(k)$ value (that is releasing lower number of vehicles from on-ramp and hence more strict ramp metering) when the current on-ramp queue length $w(k)$ is lower than the desired minimum queue length to increase the on-ramp queue length closer to the desired minimum value. The same equation will yield higher $r^{-}(k)$ when the current on-ramp queue length $w(k)$ is higher than the desired minimum queue length to reduce the on-ramp queue length closer to the desired minimum value. When $w(k)$ is equal to $W_{\min }(k)$, Equation 8 will yield $r^{\prime}(k)$ equal to $d(k-1)$, which is to maintain the existing queue length. We used $30 \%$ and $15 \%$ of the maximum queue length as activation and deactivation threshold values, respectively for the proposed control strategy. We tested values ranging from 50 to $90 \%$ for the maximum admissible queue length (a parameter value). The results for cases with a over 95\% are not presented as the on-ramp traffic that interferes with the adjacent street traffic, resulting in imprecise simulation results.

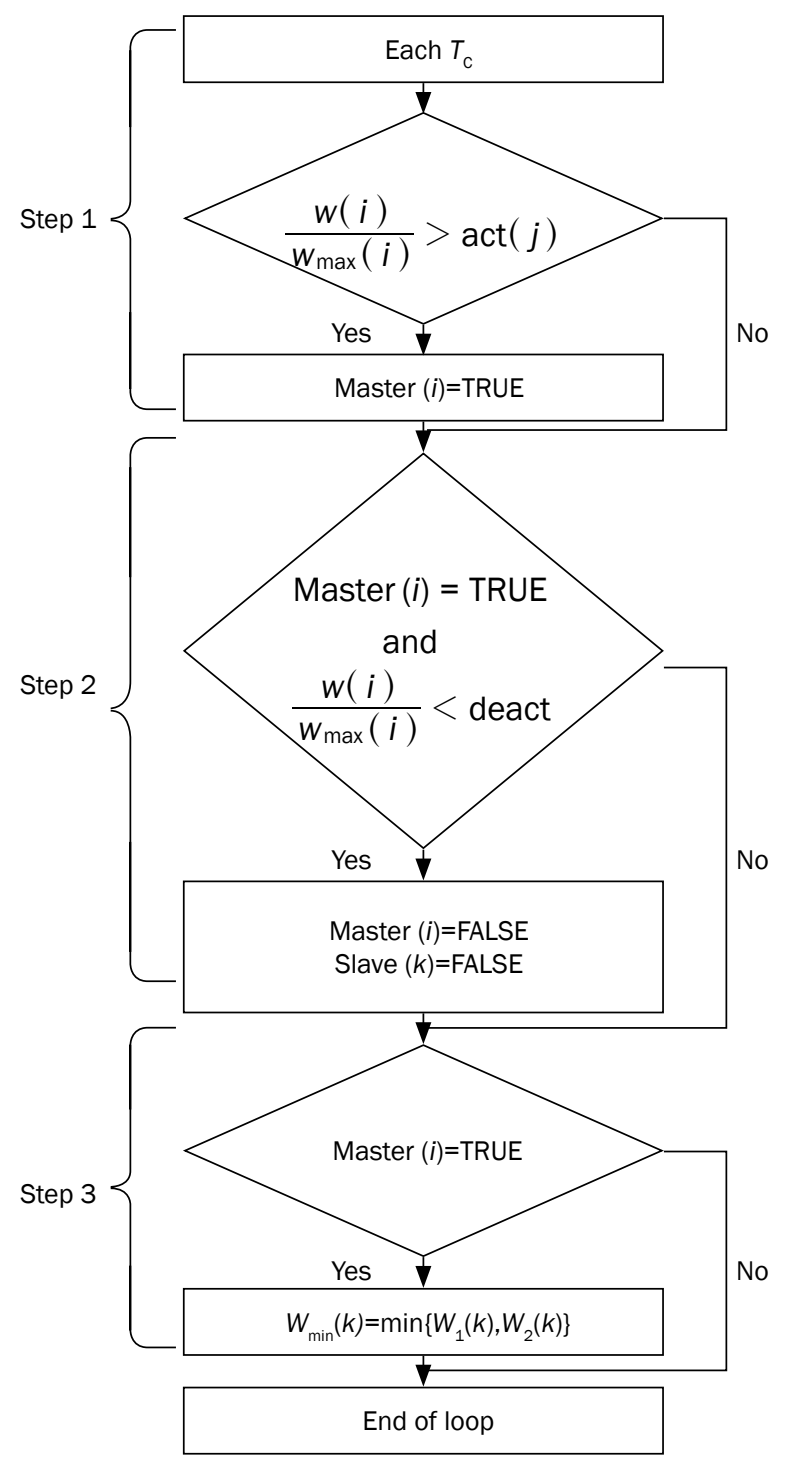

$w(i)$ is an observed queue length for an on-ramp $i$ $\operatorname{act}(j)$ is activation threshold value (30\% of the maximum queue length)

$\operatorname{deact}(j)$ is deactivation threshold value $(15 \%$ of the maximum queue length)

Figure 1 - Flowchart of HERO strategy

\section{PERFORMANCE MEASURES}

Total travel time (TTT) is widely used as a performance measure to assess system-wide efficiency performance. TTT is computed as follows: 


$$
T T T=T \sum_{K=1}^{K} \sum_{i=1}^{N} \rho_{i}(k) \Delta_{i}
$$

where: $\rho_{i}$ is density of a segment $i, T$ is measurement duration, $\Delta i$ is the distance between two measured stations $i-1$ and $i, N$ is a number of measurement stations, and $k$ is the discrete time index.

Gini coefficient, proposed by Gini [9], is used to measure the equity performance of the motorway system, which can be expressed as follows:

$$
G=\frac{1}{2 n^{2} \bar{\tau}} \sum_{i=1}^{n} \sum_{j=1}^{n}\left|\tau_{i}-\tau_{j}\right|
$$

where: $G$ is the Gini coefficient, $\bar{\tau}$ is the average delay time of all on-ramps, $\tau_{i}$ is the delay time on the $i$-th on-ramp, $\tau_{j}$ is the delay time on the $j$-th on-ramp, and $n$ is the number of on-ramps.

A zero value for Gini coefficient indicates perfect equality, while 1 indicates perfect inequality. A motorway system with the minimal TTT is considered to be the most efficient while the one with the minimal Gini coefficient is considered to be the most equitable. Thus, with the same set of constraints, optimization based on Equation 9 or 10 as the objective function to be minimized will result in efficient or equitable motorway systems respectively. Hence, it will address only one aspect of its performance. To achieve a balanced efficiency and equity gains, we can either apply a bi-objective optimization or propose a combined single index incorporating both TTT and Gini coefficient into a single objective function. We propose a combined index as follows to the combined TTT and Gini coefficient into a single objective function:

$$
\text { Combined Index }=E_{1} \cdot G+E_{2}\left(\frac{T T T_{t}-T_{T T}}{T T T_{\text {non }}}\right)
$$

where: $G$ is Gini coefficient value computed using tested control measure, TTT $_{t}$ is $T T T$ value computed using tested control measure, $T T T_{\text {non }}$ is $T T T$ value computed using no control, $T T T_{f}$ is $T T T$ value computed under free-flow condition, and $E_{1}, E_{2}$ are weight factors to stress the priority of each control objective.
Here different combinations of $E_{1}$ and $E_{2}$ will result in different control objectives. Selecting a higher value of $E_{1}$ or $E_{2}$ would lead to a more equitable (efficient) motorway system. Combined Index with different combinations of $E_{1}$ and $E_{2}$ can be useful to measure the overall performance of motorway systems from different aspects.

\section{ANALYSIS RESULTS}

A critical bottleneck section on Auckland Motorway is selected as a testbed to verify the proposed strategy. Figure 2 presents a layout of the testbed, which has 5 on-ramps and 4 off-ramps. The lengths of five on-ramps $\mathrm{O}_{1}, \mathrm{O}_{2}, \mathrm{O}_{3}, \mathrm{O}_{4}$ and $\mathrm{O}_{5}$ are recorded as 526 m, $136 \mathrm{~m}, 114 \mathrm{~m}, 315 \mathrm{~m}$ and $293 \mathrm{~m}$, respectively. A micro-simulation model of the study section is developed using AIMSUN micro-simulator [27]. The traffic data used for the model calibration and validation purpose were provided by New Zealand Transport Agency (NZTA). The data include loop detector measurements from the on-ramps, off-ramps and mainline accumulated over a 30-second time period. The data collected on Monday, $12^{\text {th }}$ March, 2012 are used to calibrate the simulation model while the data collected on Friday $9^{\text {th }}$ March 2012 are used to validate the simulation model. We developed two Application Programming Interface (API) programs in AIMSUN to implement the proposed CRM strategy and to verify the performance of the proposed strategy by comparing to the HERO strategy.

Table 1 presents TTT, Gini coefficient and the average on-ramp delay values computed for different control scenarios including no control, HERO and nine different scenarios for the proposed algorithm with the parameter a value ranging from 50\% to $90 \%$. Here no-control scenario represents a case of motorway without any type of ramp control. The highest improvements are highlighted with a dark background. HERO and all of the nine different control scenarios for the proposed algorithm yield substantial improvements compared to the no-control scenario in terms

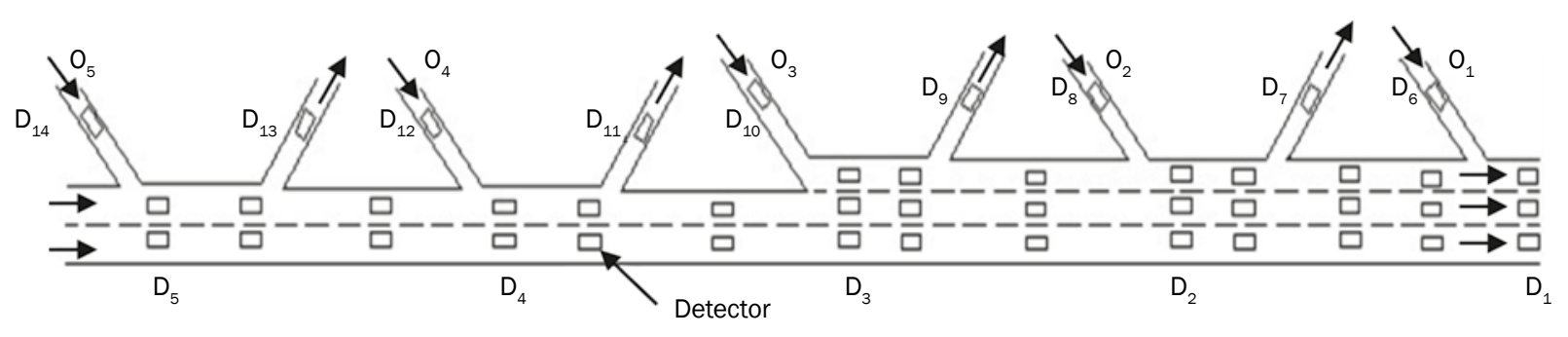

Figure 2 - Layout of the study area 
D. Li, P. Ranjitkar, Y. Zhao: Efficiency and Equity Performance of a Coordinated Ramp Metering Algorithm

Table 1 - TTT, Gini coefficient and average on-ramp delay values for different control scenarios

\begin{tabular}{||c|c|c|c|c|c|c|c|c|c||}
\hline & \multicolumn{2}{|c|}{ Total travel time [veh-h] } & \multicolumn{2}{|c|}{ Gini coefficient [\%] } & \multicolumn{3}{|c|}{ Average on-ramp delay [seconds] } \\
\hline & Value & $\%$ improvement & Value & $\%$ improvement & $\mathrm{O}_{1}$ & $\mathrm{O}_{2}$ & $\mathrm{O}_{3}$ & $\mathrm{O}_{4}$ & $\mathrm{O}_{5}$ \\
\hline No control & 1,719 & 0 & 67.26 & 0 & 274 & 33 & 3 & 3 & 3 \\
\hline HERO & 1,416 & 17.63 & 39.41 & 41.41 & 451 & 77 & 62 & 122 & 85 \\
\hline $\begin{array}{c}\text { Modified HERO } \\
(a=50 \%)\end{array}$ & 1,560 & 9.25 & 35.26 & 47.58 & 439 & 169 & 134 & 36 & 29 \\
\hline $\begin{array}{c}\text { Modified HERO } \\
(a=55 \%)\end{array}$ & 1,538 & 10.53 & 35.13 & 47.77 & 429 & 147 & 123 & 46 & 33 \\
\hline $\begin{array}{c}\text { Modified HERO } \\
(a=60 \%)\end{array}$ & 1,515 & 11.87 & 34.95 & 48.04 & 424 & 140 & 117 & 53 & 38 \\
\hline $\begin{array}{c}\text { Modified HERO } \\
(a=65 \%)\end{array}$ & 1,506 & 12.39 & 35.09 & 47.83 & 416 & 129 & 101 & 59 & 51 \\
\hline $\begin{array}{c}\text { Modified HERO } \\
(a=70 \%)\end{array}$ & 1,491 & 13.26 & 34.89 & 48.13 & 403 & 106 & 86 & 71 & 68 \\
\hline $\begin{array}{c}\text { Modified HERO } \\
(a=75 \%)\end{array}$ & 1,466 & 14.72 & 33.52 & 50.16 & 383 & 92 & 75 & 88 & 79 \\
\hline $\begin{array}{c}\text { Modified HERO } \\
(a=80 \%)\end{array}$ & 1,452 & 15.53 & 32.56 & 51.59 & 365 & 82 & 66 & 95 & 87 \\
\hline $\begin{array}{c}\text { Modified HERO } \\
(a=85 \%)\end{array}$ & 1,439 & 16.29 & 28.57 & 57.52 & 321 & 73 & 55 & 109 & 102 \\
\hline $\begin{array}{c}\text { Modified HERO } \\
(a=90 \%)\end{array}$ & 1,432 & 16.70 & 26.23 & 61.00 & 299 & 68 & 49.23 & 117 & 113 \\
\hline
\end{tabular}

of both efficiency and equity. HERO strategy yields a system-wide efficiency gain of $17.63 \%$ (measured by TTT) and records an equity gain of $40 \%$ compared to the no-control scenario. For the proposed strategy, we found that the efficiency and equity gains are sensitive towards the value of a. Here, higher value of a represents a longer desired queue length. It can be observed that the efficiency of the motorway system improves (that is, TTT value decreases) as the value of parameter a increases. The efficiency improvement reached its highest level (TTT value reduced by $16.70 \%)$ when a value is set at $90 \%$. Meanwhile, the Gini coefficient value decreases with the increase in parameter a value, which shows that in this case improvements in efficiency and equity are not conflicting as they follow a similar trend with only one exception where Gini coefficient increases from 34.95 to 35.09 when a value increases from 60 to $65 \%$. The Gini coefficient value is at its lowest (26.23\%) when a value is equal to $90 \%$, resulting in $61 \%$ improvement compared to no-control scenario while TTT value remains close to that of HERO (1,432 veh-h, an improvement of $16.7 \%$ compared to no-control scenario).

These improvements in equity performance can be attributed to a relatively fair management of queues at different on-ramps in the proposed CRM algorithm. This finding is in agreement with that of Kotsialos and Papageorgiou [24] that the storage capacity of on-ramps provided by motorways should be used to the highest degree possible as it seriously improves both efficiency and equity properties of the RM strategy. From the observations discussed earlier, it is clear that the amelioration of TTT and Gini coefficient values is highly dependent on the storage capacity of onramp; more particularly, the shortest one is the most critical for the proposed strategy.

To further investigate the trade-offs between TTT and Gini coefficient value, Figure 3 presents a scatter plot of the two variables for different a values ranging from 0.5 to 0.9 . The percentile improvements for Gini coefficient are distributed in a range from $40 \%$ to $60 \%$ the same for TTT are distributed more evenly in a range from $10 \%$ to $20 \%$. The improvement rate is positive for both variables with increasing value of a while Gini coefficient has steeper average trend than the one for TTT. Figure $3 b$ presents a scatter plot of Gini coefficient versus TTT plot for different a values. The data points show a strong correlation between the two variables in this case with a $R_{2}$ value of 0.91 and follow a non-linear (parabolic) pattern. 


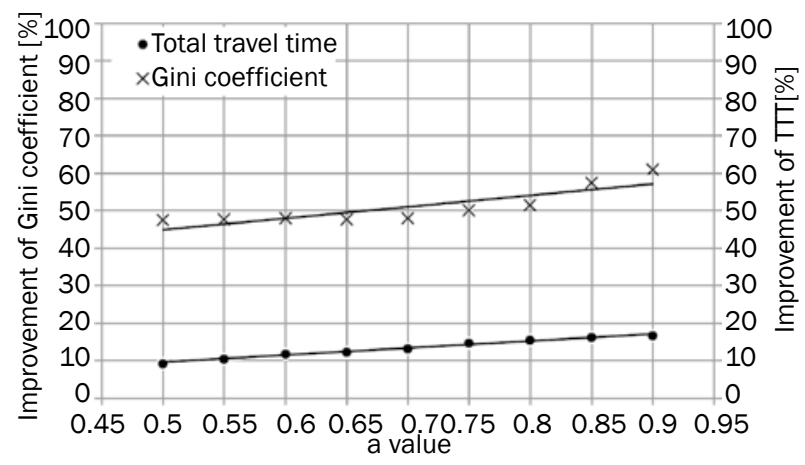

a) Percentile improvements in TTT and Gini values 40

$y=-0.001 x^{2}+3.0858 x-2313.5$

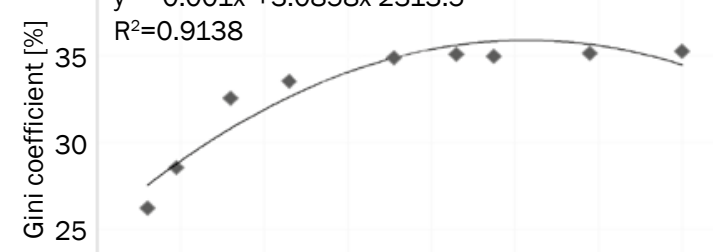
$2 0 \longdiv { 1 , 4 2 0 } 1,4401,4601,4801,5001,5201,5401,560.1,580$
TTT [veh-h]

b) Gini coefficient versus TTT scatter plot

Figure 3 - Scatter plots of TTT and Gini coefficient for different a values for the proposed strategy
Figure 4 presents the density contour plots along the mainline of the controlled segments for four control scenarios including no-control, HERO and two scenarios for the proposed strategy with parameter a taking values of 0.5 and 0.9 . It can be observed that the density exceeds $80 \mathrm{veh} / \mathrm{km}$ during the peak hours starting from 6:30 a.m. near the first detector zone $D_{1}$, which is close to the first on-ramp $\mathrm{O}_{1}$. The shockwave travels to the successive segments and reaches up to detector zone $\mathrm{D}_{4}$. For HERO control strategy, the density at $D_{1}$ remains close to $80 \mathrm{veh} / \mathrm{km}$ while for successive segments it seldom exceeds $60 \mathrm{veh} / \mathrm{km}$. For the proposed strategy with a value of 0.5 , the density at the first three detector zones $D_{1}$ to $D_{3}$ reaches close to $80 \mathrm{veh} / \mathrm{km}$ during peak hours. For the proposed strategy with a value of 0.9 , the density remains below 80 veh/ $\mathrm{km}$ at the first detector zone $D_{1}$ and for successive zones it seldom exceeds $60 \mathrm{veh} / \mathrm{km}$. The density looks more evenly distributed in the controlled segments for the proposed algorithm with an a value of 0.9 compared to the other three control scenarios.

Figure 5 presents the average delay at five on-ramps under different control scenarios. Travel delay is the extra travel time beyond free flow conditions. Average on-ramp delay values can be directly extracted from AINSUM simulator. $\mathrm{O}_{1}$ (the master on-ramp), which is the closest to the critical bottleneck experiences the
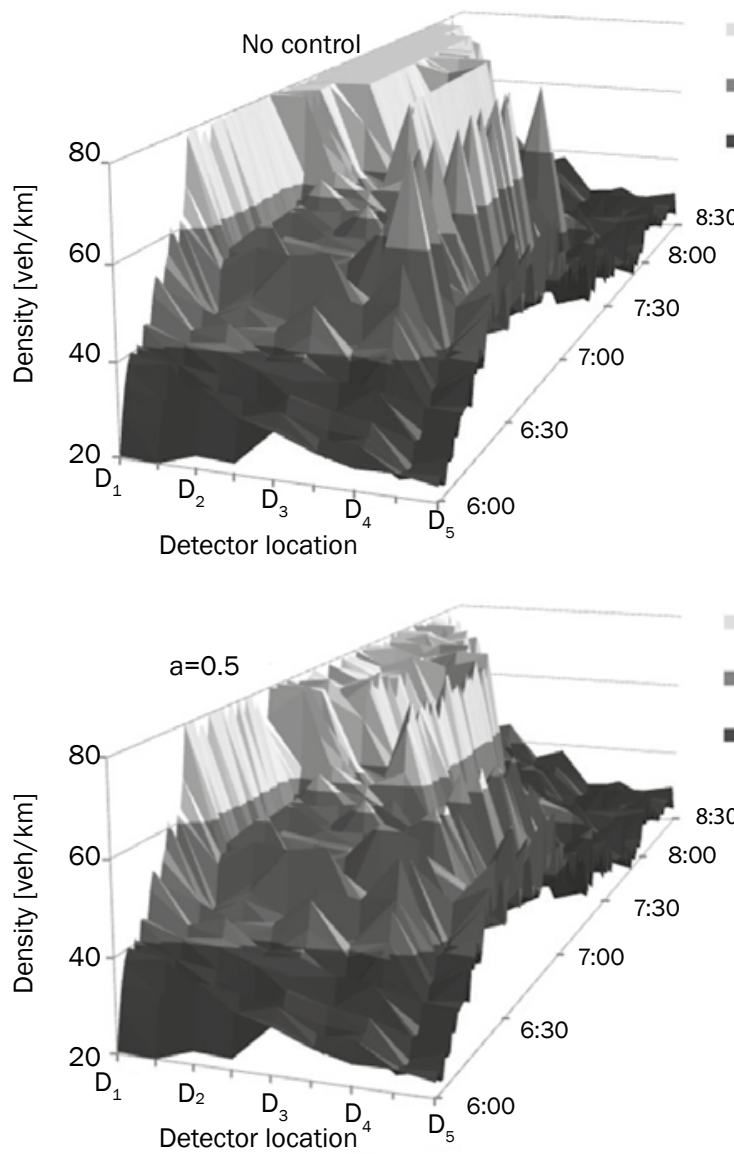
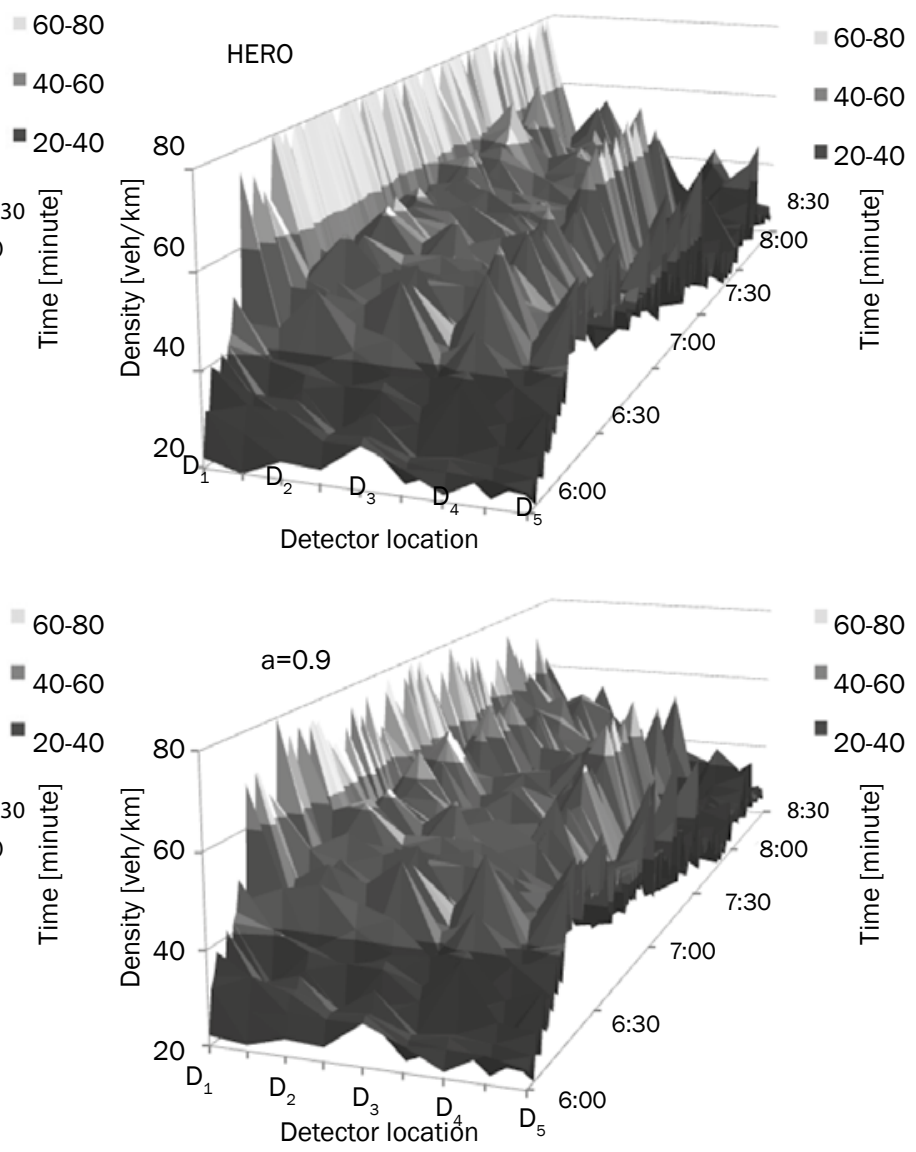

Figure 4 - Density contour plots for different control scenarios 
longest delay and $\mathrm{O}_{5}$, which is located farthest from the critical bottleneck, experiences the minimum delay. Although the queue length formed at $\mathrm{O}_{2}$ and $\mathrm{O}_{3}$ discussed earlier are shorter than $\mathrm{O}_{4}$ and $\mathrm{O}_{5}$, the average delay is comparable at these on-ramps. Higher a value yields lower average delay for the first three on-ramps while the results are opposite for the last two on-ramps $\mathrm{O}_{4}$ and $\mathrm{O}_{5}$. No control scenario yields the lowest average delay among all the scenarios; this might be due to the absence of RM control. For HERO control strategy, the average on-ramp delay is less spread than the modified strategy cases, representing a less equitable system.

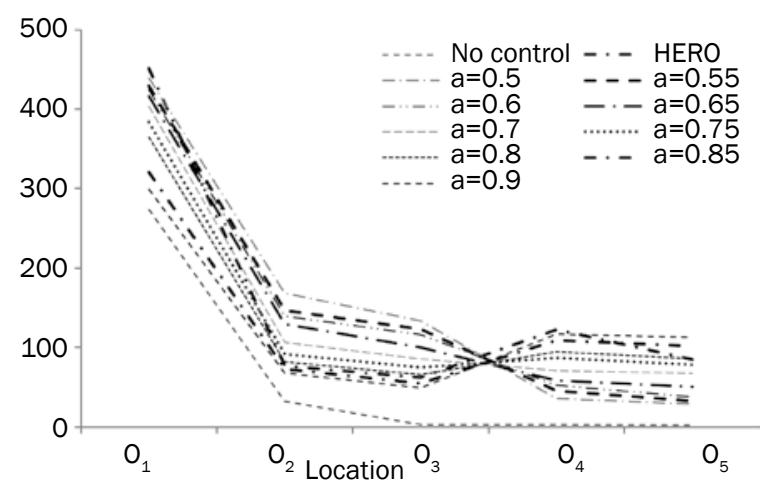

Figure 5 - Average on-ramp delay for different control scenarios

Figure 6 presents the values of combined index computed for tested scenarios using $E_{1}=1$ and $E_{2}=1$. Here no-control scenario witnesses the highest combined index value of 1.15, representing its worst performance in terms of both efficiency and equity among all the tested control scenarios. The proposed control strategies with a value ranging from $70 \%$ to $90 \%$ have outperformed the HERO strategy in terms of a balanced efficiency and equity gains. The proposed control strategy with a value of 0.9 (or $90 \%$ ) yields the lowest combined index value, representing the most efficient-equitable motorway system among all the tested control scenarios.

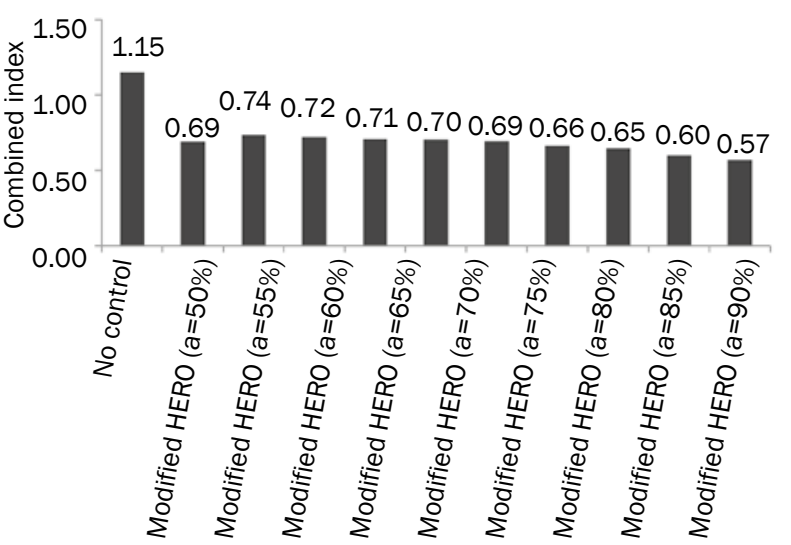

Figure 6 - Combined index values for different control scenarios

\section{CONCLUSION}

This paper proposes a coordinated ramp metering (CRM) algorithm to achieve an efficient as well as equitable motorway system. HEuristic Ramp metering coOrdination (HERO) control strategy is modified to improve its equity performance. Total travel time is used to measure the efficiency while Gini coefficient is used to measure equity. A combined index is proposed incorporating the two measures to serve as an objective function to solve the bi-objective control design problem. The performance of the proposed strategy is verified by comparison against a well-established coordinated RM strategy HERO.

The main conclusions that can be drawn from this study can be summarised as follows:

- The proposed CRM algorithm shows prospects to improve significantly the equity performance of the motorway system while maintaining nearly the same level of efficiency to that of the HERO strategy.

- The proposed combined index can be useful to achieve a balanced efficiency and equity gains for the motorway system.

It shall be noted that findings of this study are limited in scope as they are obtained on the basis of a particular motorway section for a representative day. One suggestion for the future studies is to perform similar studies for various motorway networks before generalizing any such findings. Furthermore, in this study all the parameters in the proposed modified HERO strategy are optimized manually for the testbed in Auckland. However, a modified HERO strategy with the pre-specified setting parameters cannot adequately deal with the disturbances (such as inconsistent traffic demand pattern) and changes in traffic system (weather condition, incidents or road maintenance). Therefore, future works around this research should aim at using optimization methods (e.g., Genetic Algorithm) to adaptively tune the parameters of the proposed strategy for application in other motorway networks.

李多 博士 (通讯作者)

公路学院, 长安大学, 西安市, 陕西省, 中国

电子邮箱：duoli0725@gmail. com

PRAKASH RANJITKAR, 博士

土木与环境工程学院, 奥克兰大学, 奥克兰, 新西兰 电子邮箱: p. ranjitkar@auckland. ac. nz 赵一飞, 博士

公路学院, 长安大学, 西安市, 陕西省, 中国

电子邮箱：409291742@qq. com

\section{多匝道协同控制方法的通行能力及用户平衡性研究}

\section{李多}

博士, 现为长安大学公路学院讲师。本科毕业于华 中科技大学交通工程专业, 硕士毕业于澳大利亚昆 
士兰大学交通工程专业，博士毕业于新西兰奥克兰 大学交通工程专业。主要从事高速公路及城市快速 路交通组织与管理，交通安全，信号控制，智能交 通系统，事故控制系统，交通仿真与建模，智能算 法等方面的教学与研究工作。

\section{关键词}

信号控制，智能交通系统，交通组织与管理，交通 安全, 交通仿真, 智能算法

\section{REFERENCES}

[1] Zhang L, Levinson D. Balancing Efficiency and Equity of Ramp Meters. ASCE Journal of Transportation Engineering. 2005;131(6):477-481.

[2] Papageorgiou M, Papamichail I. Handbook of Ramp Metering. Document for European RAmp Metering Project (EURAMP); 2007.

[3] Li D, Ranjitkar P. Assessing Ramp Metering and Variable Speed Limits Strategies for Auckland Motorway, Journal of Eastern Asia Society for Transportation Studies. 2013;10:1856-1871.

[4] Li D, Ranjitkar P. Performance Evaluation of Ramp Metering Algorithms Combined with Variable Speed Limits for Auckland Motorway. 92nd Annual Meeting of Transportation Research Board; 2013 Jan 13-17; Washington DC; 2013.

[5] Pinnell C, Drew D, McCasland W, Wattleworth I. Evaluation of entrance ramp control on a six-mile freeway section. Highway Research Record. 1967;157:22-76.

[6] Yin Y, Liu H, Benouar H. A Note on Equity of Ramp Metering. 7th IEEE Intelligent Transportation Systems Conference; 2000 Oct 1-3; Washington, DC; 2000.

[7] Levinson D, Zhang L, Das S, Sheikh A. Evaluating Ramp Meter: Evidence from the Twin Cities Ramp Meter Shut-off. 81st Annual Meeting of Transportation Research Board; 2002 Jan 13-17; Washington, DC; 2002.

[8] Gini C. On the measure of concentration with especial reference to income and wealth. Cowles Commission; 1936.

[9] Kotsialos A, Papageorgiou M, Middelham F. Optimal Coordinated Ramp Metering with Advanced Motorway Optimal Control. Transportation Research Record. 2001;1748:55-65.

[10] Zhang L, Levinson D. Optimal Freeway Ramp Control Without Origin-destination Information. Transportation Research B. 2004;38:869-887.

[11] Zhang HM, Shen W. Access Control Policies without Inside Queues: Their Properties and Public Policy Implications. Transportation Research B. 2010;44: 132-1147.
[12] Papamichail I, Papageorgiou M. Traffic-responsive Linked Ramp Metering Control. IEEE Transactions on Intelligent Transportation Systems. 2008;9:111-121.

[13] Papamichail I, Papageorgiou M, Vong V, Gaffney J. Heuristic Ramp-Metering Coordinated Strategy Implemented at the Monash Freeway. Transportation Research Record. 2010;2178:10-20.

[14] Minnesota Department of Transportation. Stratified Zone Metering-The Minnesota Algorithm. MnDOT, Minnesota; 2003.

[15] Li D, Ranjitkar P, Ceder A. An Integrated Approach to Combine Ramp Metering and Variable Speed Limits to Improve Motorway Performance. Transportation Research Record. 2014;2470:86-94.

[16] Li D, Ranjitkar P. A Fuzzy Logic-based Variable Speed Limit Controller. Journal of Advanced Transportation. 2015;49(8):913-927.

[17] Lakshmanan T, Nijkamp P, Rietveld P, Verhoef E. Benefits and Costs of Transport: Classification, Methodologies and Policies. Regional Science. 2001;80: 139-164.

[18] Hasan M, Ha M, Ben-Akiva M. Evaluation of Ramp Control Algorithms Using Microscopic Traffic Simulation. Transportation Research Part C. 2002;10(c):229-256.

[19] Papageorgiou M, Haj-Salem H, Middclham F. ALINEA Local Ramp Metering: Summary of Field Results. Transportation Research Record. 1997;1603:90-98.

[20] Kesten A, Ergün M, Yai T. An Analysis on Efficiency and Equity of Fixed-Time Ramp Metering. Journal of Transportation Technologies. 2013;3:48-56.

[21] Zhang L, Levinson D. Balancing efficiency and equity of ramp meters. 82nd Annual Meeting of Transportation Research Board; 2003 Jan 12-16; Washington DC; 2003.

[22] Jacobson L, Henry K, Mahyar O. Real-time Metering Algorithm for Centralized Control. Transportation Research Record. 1989;1232:17-26.

[23] Kotsialos A, Papageorgiou M. Efficiency and Equity Properties of Freeway Network-wide Ramp Metering with AMOC. Transportation Research Part C. 2003;12:401-420.

[24] Lorenz MO. Methods of Measuring the Concentration of Wealth. Publication of the American Statistical Association; 1905.

[25] Levinson D, Zhang L, Das S, Sheikh A. Ramp meters on trial: evidence from the Twin Cities ramp meters shutoff. 81st Annual Meeting of Transportation Research Board; 2002 Jan 13-17; Washington, DC; 2002.

[26] Papageorgiou M, Hadj-Salem H, Blosseville JM. ALINEA: a Local Feedback Control Law for Onramp Metering. Transportation Research Record. 1991;1320: 58-64.

[27] TSS. AIMSUN 7 Dynamic Simulators User`s Manual. Transport Simulation Systems; 2012. 